\title{
shadow: R Package for Geometric Shadow Calculations in an Urban Environment
}

\author{
by Michael Dorman, Evyatar Erell, Adi Vulkan, Itai Kloog
}

\begin{abstract}
This paper introduces the shadow package for R. The package provides functions for shadowrelated calculations in the urban environment, namely shadow height, shadow footprint and Sky View Factor (SVF) calculations, as well as a wrapper function to estimate solar radiation while taking shadow effects into account. All functions operate on a layer of polygons with a height attribute, also known as "extruded polygons" or 2.5D vector data. Such data are associated with accuracy limitations in representing urban environments. However, unlike 3D models, polygonal layers of building outlines along with their height are abundantly available and their processing does not require specialized closed-source 3D software. The present package thus brings spatio-temporal shadow, SVF and solar radiation calculation capabilities to the open-source spatial analysis workflow in R. Package functionality is demonstrated using small reproducible examples for each function. Wider potential use cases include urban environment applications such as evaluation of micro-climatic influence for urban planning, studying urban climatic comfort and estimating photovoltaic energy production potential.
\end{abstract}

\section{Introduction}

Spatial analysis of the urban environment (Biljecki et al., 2015) frequently requires estimating whether a given point is shaded or not, given a representation of spatial obstacles (e.g. buildings) and a time-stamp with its associated solar position. For example, we may be interested in -

- Calculating the amount of time a given roof or facade is shaded, to determine the utility of installing photovoltaic cells for electricity production (e.g. Redweik et al., 2013).

- Calculating shadow footprint on vegetated areas, to determine the expected influence of a tall new building on the surrounding microclimate (e.g. Bourbia and Boucheriba, 2010).

Such calculations are usually carried out using GIS-based models (Freitas et al., 2015), in either vector-based $3 \mathrm{D}$ or raster-based $2.5 \mathrm{D}$ settings. Both approaches have their advantages and limitations, as discussed in the following paragraphs.

Shadow calculations on vector-based 3D models of the urban environment are mostly restricted to proprietary closed-source software such as ArcGIS (ESRI, 2017) or SketchUp (Google, 2017), though recently some open-source models such as SURFSUN3D have been developed (Liang et al., 2015). One of the drawbacks of using closed-source software in this context is the difficulty of adjusting the software for specific needs and uncommon scenarios. This problem is especially acute in research settings, where flexibility and extensibility are essential for exploring new computational approaches. The other difficulty with using 3D software in urban spatial analysis concerns interoperability of file formats. Since ordinary vector spatial data formats, such as the ESRI Shapefile, cannot represent three-dimensional surfaces, 3D software is associated with specialized file formats. The latter cannot be readily imported to a general-purpose geocomputational environment such as $\mathrm{R}$ or Python (Van Rossum and Drake, 2011), thus fragmenting the analysis workflow. Moreover, most 3D software, such as those mentioned above, are design-oriented, thus providing advanced visualization capabilities but limited quantitative tools (calculating areas, angles, coordinates, etc.). Finally, true-3D databases of large urban areas are difficult to obtain, while vector-based 2.5D databases (building outline and height, see below) are almost universal. The advantages of true-3D software are "wasted" when the input data are $2.5 \mathrm{D}$, while the disadvantages, such as lack of quantitative procedures and data interoperability difficulties, still remain.

Raster-based 2.5D solutions, operating on a Digital Elevation Model (DEM) raster, are much simpler and have thus been more widely implemented in various software for several decades (Kumar et al., 1997; Ratti and Richens, 2004). For example, raster-based shadow calculations are available in open-source software such as the r.sun command (Hofierka and Suri, 2002) in GRASS GIS (GRASS Development Team, 2017), the UMEP plugin (Lindberg et al., 2018) for QGIS (QGIS Development Team, 2017) and package insol (Corripio, 2014) in R. In the proprietary ArcGIS software, raster-based shadow calculations are provided through the Solar Analyst extension (Fu and Rich, 1999). Thanks to this variety of tools, raster-based shadow modelling can be easily incorporated within a general 
spatial analysis workflow. However, raster-based models are more suitable for large-scale analysis of natural terrain, rather than fine-scale urban environments, for the following reasons -

- A raster representing surface elevation, known as a DEM, at sufficiently high resolution for the urban context, may not be available and is expensive to produce, e.g. using airborne Light Detection And Ranging (LiDAR) surveys (e.g. Redweik et al., 2013). Much more commonly, municipalities and other sources such as OpenStreetMap (Haklay and Weber, 2008) offer 2.5D vector-based data on cities, i.e. polygonal layers of building outlines associated with height attributes.

- Rasters are composed of pixels, which have no natural association to specific urban elements, such as an individual building, thus making it more difficult to associate analysis results with the corresponding urban elements.

- Vertical surfaces, such as building facades, are rare in natural terrain yet very common in urban environments. Raster-based representation of facades is problematic since the latter correspond to (vertical) discontinuities in the 2.5D digital elevation model, requiring unintuitive workarounds (Redweik et al., 2013).

It should be noted that more specialized approaches have been recently developed to address some of the above-mentioned difficulties, but they are usually not available as software packages (e.g. Redweik et al., 2013; Hofierka and Zlocha, 2012).

The shadow package (Dorman, 2019) aims at addressing these limitations by introducing a simple $2.5 \mathrm{D}$ vector-based algorithm for calculating shadows, Sky View Factor (SVF) and solar radiation estimates in the urban environment. The algorithms operate on a polygonal layer extruded to 2.5D, also known as Levels-of-Detail (LoD) 1 in the terminology of the CityGML standard (Gröger and Plümer, 2012). On the one hand, the advantages of individual urban element representation (over raster-based approach) and input data availability (over both raster-based and full 3D approaches) are maintained. On the other hand, the drawbacks of closed-source software and difficult interoperability (as opposed to full 3D environment) are avoided.

As demonstrated below, functions in the shadow package operate on a vector layer of obstacle outlines (e.g. buildings) along with their heights, passed as a "SpatialPolygonsDataFrame" object defined in package sp (Bivand et al., 2013; Pebesma and Bivand, 2005). The latter makes incorporating shadow calculations in Spatial analysis workflow in R straightforward. Functions to calculate shadow height, shadow ground footprint, Sky View Factor (SVF) and solar radiation are implemented in the package.

\section{Theory}

\section{Shadow height}

All functions currently included in package shadow are based on trigonometric relations in the triangle defined by the sun's rays, the ground - or a plane parallel to the ground - and an obstacle.

For example, shadow height at any given ground point can be calculated based on (1) sun elevation, (2) the height of the building(s) that stand in the way of sun rays and (3) the distance(s) between the queried point and the building(s) along the sun rays projection on the ground. Figure 1 depicts a scenario where shadow is being cast by building $A$ onto the facade of building $B$, given the solar position defined by its elevation angle $\alpha_{\text {elev }}$ and azimuth angle $\alpha_{a z}$. Once the intersection point is identified (marked with $\mathrm{x}$ in Figure 1$)$, shadow height $\left(h_{\text {shadow }}\right)$ at the queried point (viewer) can be calculated based on (1) sun elevation $\left(\alpha_{\text {elev }}\right)$, (2) the height of building A $\left(h_{\text {build }}\right)$ and (3) the distance $\left(\right.$ dist $\left._{1}\right)$ between the viewer and intersection point $x$ (Equation 1$)$.

$$
h_{\text {shadow }}=h_{\text {build }}-\text { dist }_{1} \cdot \tan \left(\alpha_{\text {elev }}\right)
$$

The latter approach can be extended to the general case of shadow height calculation at any ground location and given any configuration of obstacles. For example, if there is more than one obstacle potentially casting shadow on the queried location, we can calculate $h_{\text {shadow }}$ for each obstacle and then take the maximum value.

\section{Logical shadow flag}

Once the shadow height is determined, we may evaluate whether any given 3D point is in shadow or not. This is done simply by comparing the Z-coordinate (i.e. height) of the queried point with the calculated shadow height at the same $X-Y$ (i.e. ground) location. 


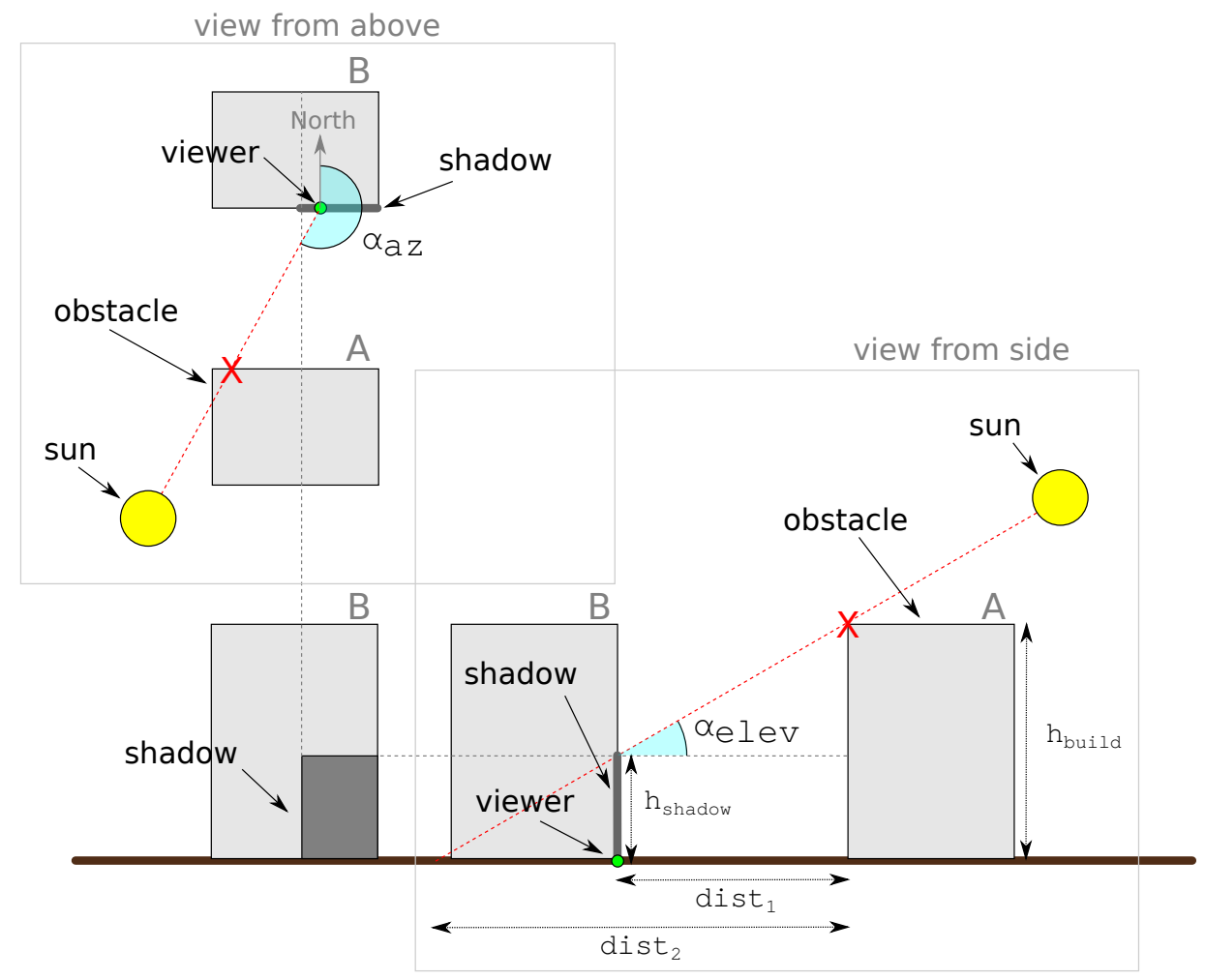

Figure 1: Shadow height calculation

\section{Shadow footprint}

Instead of calculating shadow height at a pre-specified point (e.g. the viewer in Figure 1), we can set $h_{\text {shadow }}$ to zero and calculate the distance $\left(\right.$ dist $\left._{2}\right)$ where the shadow intersects ground level (Equation 2).

$$
\text { dist }_{2}=\frac{h_{\text {build }}}{\tan \left(\alpha_{\text {elev }}\right)}
$$

Shifting the obstacle outline by the resulting distance $\left(\right.$ dist $\left._{2}\right)$ in a direction opposite to sun azimuth $\left(\alpha_{a z}\right)$ yields a shadow footprint outline (Weisthal, 2014). Shadow footprints are useful to calculate the exact ground area that is shaded at specific time. For example, Figure 2 shows the shadow footprints produced by a single building at different times of a given day.

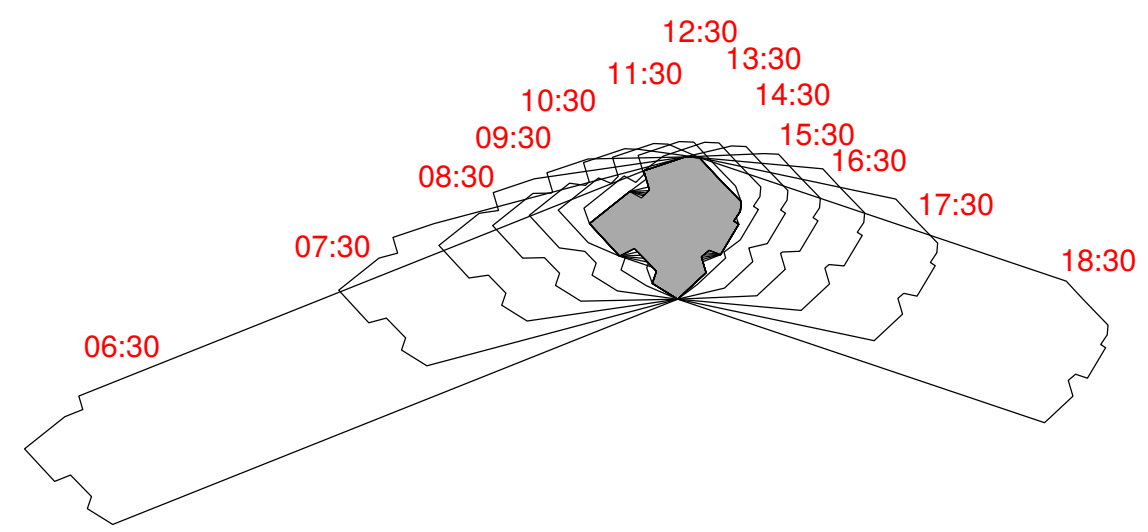

Figure 2: Shadow footprints cast by a building on a horizontal ground surface at hourly intervals on 2004-06-24. The building, indicated by the gray shaded area, is located at $31.97^{\circ} \mathrm{N} 34.78^{\circ} \mathrm{E}$, and is 21.38 meters tall 
view from above

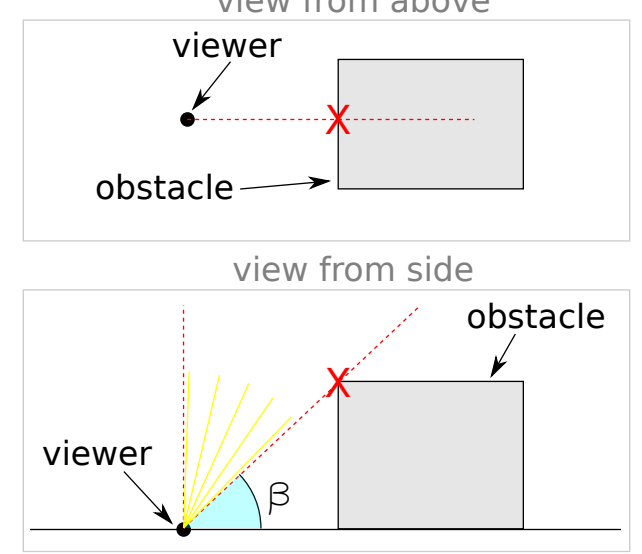

Figure 3: Sky View factor calculation

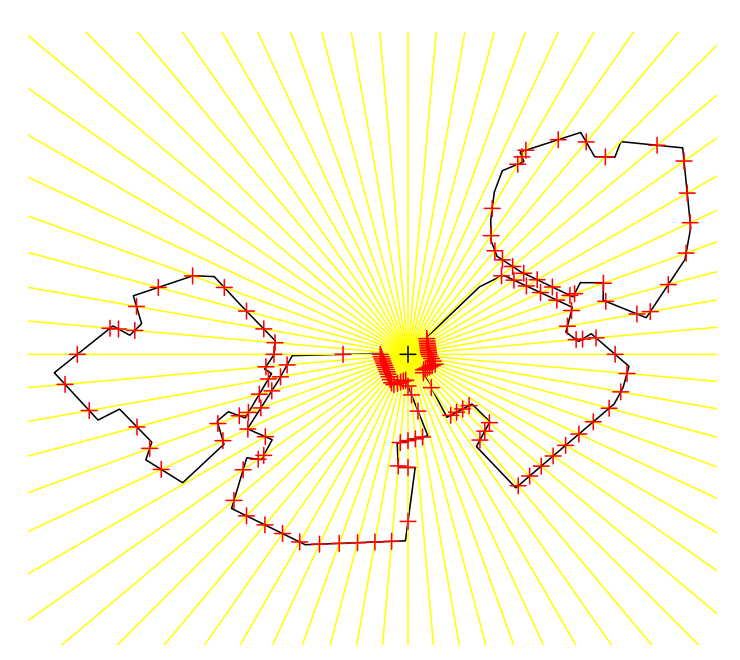

Figure 4: Angular cross sections for calculating the Sky View Factor (SVF)

\section{Sky View Factor (SVF)}

The Sky View Factor (Beckers, 2013; Erell et al., 2011; Grimmond et al., 2001) is the extent of sky observed from a point as a proportion of the entire sky hemisphere. The SVF can be calculated based on the maximal angles $(\beta)$ formed in triangles defined by the queried location and the obstacles (Figure $3)$, evaluated in multiple circular cross-sections surrounding the queried location. Once the maximal angle $\beta_{i}$ is determined for a given angular section $i, S V F_{i}$ for that particular section is defined (Gál and Unger, 2014) in Equation 3.

$$
S V F_{i}=1-\sin ^{2}\left(\beta_{i}\right)
$$

For example, in case $\left(\beta_{i}=45^{\circ}\right)$, as depicted in Figure $3, S V F_{i}$ is equal to -

$$
S V F_{i}=1-\sin ^{2}\left(45^{\circ}\right)=0.5
$$

Averaging $S V F_{i}$ values for all $i=1,2, \ldots, n$ circular cross-sections gives the final $S V F$ estimate for the queried location (Equation 4).

$$
S V F=\frac{\sum_{i=1}^{n} S V F_{i}}{n}
$$

The number of evaluated cross sections depends on the chosen angular resolution. For example, an angular resolution of $5^{\circ}$ means the number of cross sections is $n=360^{\circ} / 5^{\circ}=72$ (Figure 4). 


\section{Solar radiation}

\section{Components}

Frequently, evaluating whether a given location is shaded, and when, is just a first step towards evaluating the amount of solar radiation for a given period of time. The annual insolation at a given point is naturally affected by the degree of shading throughout the year, but shading is not the only factor.

The three components of the solar radiation are the direct, diffuse and reflected radiation -

- Direct radiation refers to solar radiation traveling on a straight line from the sun to the surface of the earth. Direct radiation can be estimated by taking into account: (1) shading, (2) surface orientation relatively to the sun, and (3) meteorological measurements of direct radiation on a horizontal plane or on a plane normal to the beam of sunlight.

- Diffuse radiation refers to solar radiation reaching the Earth's surface after having been scattered from the direct solar beam by molecules or particulates in the atmosphere. Diffuse radiation can be estimated by taking into account: (1) SVF, and (2) meteorological measurements of diffuse radiation at an exposed location.

- Reflected radiation refers to the sunlight that has been reflected off non-atmospheric obstacles such as ground surface cover or buildings. Most urban surfaces have a low albedo: asphalt reflects only 5-10 percent of incident solar radiation, brick and masonry 20-30 percent, and vegetation about 20 percent. Because a dense urban neighborhood will typically experience multiple reflections, an iterative process is required for a complete analysis. Calculating reflected radiation requires taking into account reflective properties of the various surfaces, their geometrical arrangement (Givoni, 1998) and their view factors from the receiving surface, which is beyond the scope of the shadow package.

The diffuse radiation component is the dominant one on overcast days, when most radiation is scattered, while the direct radiation component is dominant under clear sky conditions when direct radiation reaches the earth's surface.

\section{Direct Normal Irradiance}

Equation 5 specifies the Coefficient of Direct Normal Irradiance for a vertical facade surface, as function of solar position given by the difference between facade azimuth and sun azimuth angles, and sun elevation angle, at time $t$.

$$
\theta_{\text {facade }, t}=\cos \left(\alpha_{a z, t}-\alpha_{a z}^{\prime}\right) \cdot \cos \left(\alpha_{\text {elev }, t}\right)
$$

In Equation $5, \theta_{\text {facade }, t}$ is the Coefficient of Direct Normal Irradiance on a facade at time $t, \alpha_{a z, t}$ is the sun azimuth angle at time $t$ (see Figure 1 ), $\alpha_{a z}^{\prime}$ is the facade azimuth angle, i.e. the direction where the facade is facing, and $\alpha_{\text {elev, } t}$ is sun elevation angle at time $t$ (see Figure 1 ). Note that all of latter variables, with the exception of facade azimuth angle $\alpha_{a z}^{\prime}$, are specific for the time interval $t$ due to the variation in solar position.

Horizontal roof surfaces, unlike facades, are not tilted towards any particular azimuth ${ }^{1}$. Equation 5 thus simplifies to Equation 6 when referring to a roof, rather than a facade, surface.

$$
\theta_{\text {roof }, t}=\cos \left(90^{\circ}-\alpha_{\text {elev }, t}\right)
$$

Figure 5 demonstrates the relation given in Equations 5 and 6 for the entire relevant range of solar positions relative to facade or roof orientation. Again, note that for roof surfaces, the $\theta_{\text {roof, } t}$ coefficient is only dependent on sun elevation angle $\alpha_{\text {elev, }}$ (Equation 6) as illustrated on the right panel of Figure 5. (The code for producing Figure 5 can be found in the help page of function coefDirect from shadow).

For example, the left panel in Figure 5 shows that maximal proportion of incoming solar radiation (i.e. $\theta_{\text {facade }, t}=1$ ) on a facade surface is attained when facade azimuth is equal to sun azimuth and sun elevation is $0\left(\alpha_{\text {elev,t }}=0^{\circ}\right.$, i.e. facade directly facing the sun). Similarly, the right panel shows that maximal proportion of solar radiation on a roof surface (i.e. $\theta_{\text {roof }, t}=1$ ) is attained when the sun is at the zenith $\left(\alpha_{\text {elev }, t}=90^{\circ}\right.$, i.e. sun directly above the roof).

Once the Coefficient of Direct Normal Irradiance $\theta_{\text {facade }, t}$ or $\theta_{\text {roof }, t}$ is determined, the Direct Normal Irradiance meteorological measurement $\mathrm{rad}_{\text {direct, } t}$ referring to the same time interval $t$, usually on an

\footnotetext{
${ }^{1}$ It should be noted that roof surfaces may be pitched rather than horizontal; however 2.5D models, which shadow supports, can only represent horizontal roofs
} 
Facade

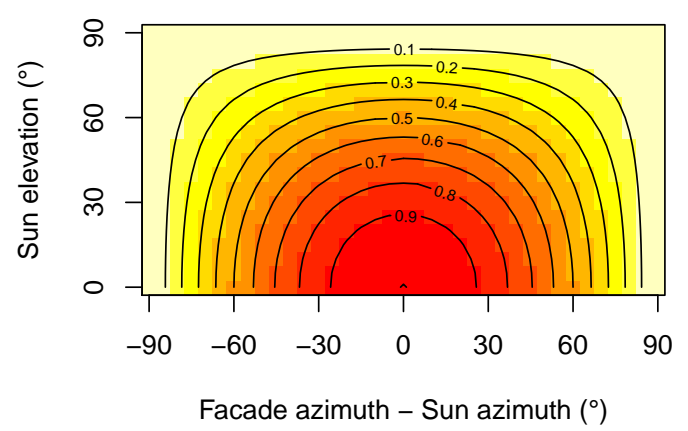

Roof

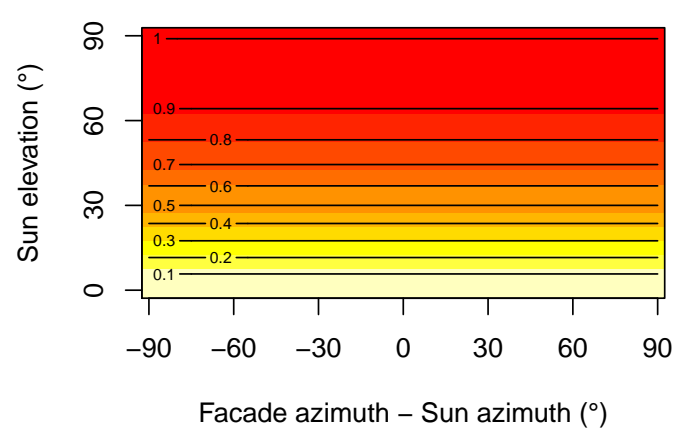

Figure 5: Coefficient of Direct Normal Irradiance, as function of solar position, expressed as the difference between facade and sun azimuths (X-axis) and sun elevation (Y-axis). The left panel refers to a facade, the right panel refers to a roof. Note that a horizontal roof has no azimuth, thus the $\mathrm{X}$-axis is irrelevant for the right panel and only shown for uniformity

hourly time step, is multiplied by the coefficient at a point on the building surface to give the local irradiation at that point (Equation 7). The result $\mathrm{rad}_{\text {direct,t }}^{\prime}$ is the corrected Direct Irradiance the surface receives given its orientation relative to the solar position.

$$
\mathrm{rad}_{\text {direct,t }}^{\prime}=\theta_{t} \cdot \mathrm{rad}_{\text {direct }, t}
$$

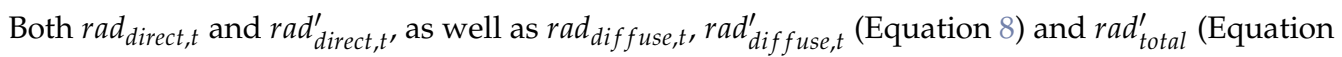
9) (see below), are given for each time interval $t$ in units of power per unit area, such as $\mathrm{kWh} / \mathrm{m}^{2}$.

\section{Diffuse Horizontal Irradiance}

Moving on to discussing the second component in the radiation balance, the diffuse irradiance. Diffuse irradiance is given by the meteorological measurement of Diffuse Horizontal Irradiance rad $_{\text {diffuse,t }}$, which needs to be corrected for the specific proportion of viewed sky given surrounding obstacles expressed by SVF. Assuming isotropic contribution (Freitas et al., 2015), rad diffuse,t is the corrected diffuse irradiance the surface receives (Equation 8). Note that $S V F$ is unrelated to solar position; it is a function of the given configuration of the queried location and surrounding obstacles, and is thus invariable for all time intervals $t$.

$$
\operatorname{rad}_{\text {diffuse,t }}^{\prime}=S V F \cdot \operatorname{rad}_{\text {diffuse,t }}
$$

\section{Total irradiance}

Finally, the direct and diffuse radiation estimates are summed for all time intervals $t$ to obtain the total (e.g. annual) insolation for the given surface $\mathrm{rad}_{\text {total }}^{\prime}$ (Equation 9). The sum refers to $n$ intervals $t=1,2, \ldots, n$, commonly $n=24 \times 365=8,760$ when referring to an annual radiation estimate using an hourly time step.

$$
\operatorname{rad}_{\text {total }}^{\prime}=\sum_{t=1}^{n} \operatorname{rad}_{\text {direct }, t}^{\prime}+\sum_{t=1}^{n} \operatorname{rad}_{\text {diffuse }, t}^{\prime}
$$

\section{Package structure}

The shadow package contains four "low-level" functions, one "high-level" function, and several "helper functions".

The "low-level" functions calculate distinct aspects of shading, and the SVF -

- shadowHeight - Calculates shadow height

- inshadow - Determines a logical shadow flag (in shadow or not)

- shadowFootprint - Calculates shadow footprint

- SVF - Calculates the SVF 


\begin{tabular}{l|c|c|c|c}
\hline Function & Location & Obstacles & Sun Pos. & Output \\
\hline shadowHeight & Points (2D) / Raster & Polygons & Matrix & Numeric matrix / Raster \\
inShadow & Points (2D/3D) / Raster & Polygons & Matrix & Logical matrix / Raster \\
shadowFootprint & - & Polygons & Matrix & Polygons \\
SVF & Points (2D/3D) / Raster & Polygons & - & Numeric vector / Raster \\
\hline
\end{tabular}

Table 1: Inputs and outputs for main functions in package shadow

Table 1 gives a summary of the (main) input and output object types for each of the "low-level" functions. The following list clarifies the exact object classes referenced in the table -

- The queried locations points (e.g. the viewer point in Figure 1) can be specified in several ways. Points ("SpatialPoints*") can be either 2D, specifying ground locations, or $3 \mathrm{D}^{2}$ - specifying any location on the ground or above ground. Alternatively, a raster ("Raster*") can be used to specify a regular grid of ground locations. Note that the shadow height calculation only makes sense for ground locations, as height above ground is what the function calculates, so it is not applicable for 3D points

- The obstacle polygons are specified as a "SpatialPolygonsDataFrame" object having a height attribute ("extrusion" height) given in the same units as the layer Coordinate Reference System (CRS), usually meters. Geographic coordinates (long/lat) are not allowed because these units are meaningless for specifying height

- Solar position matrix is given as a "matrix" object, where the first column specifies sun azimuth angle and the second column specifies sun elevation angle. Both angles should be given in decimal degrees, where -

- sun azimuth (e.g. $\alpha_{a z}$ in Figure 1) is measured clockwise relative to North, i.e North $=0^{\circ}$, East $=90^{\circ}$, South $=180^{\circ}$, West $=270^{\circ}$

- sun elevation (e.g. $\alpha_{\text {elev }}$ in Figure 1) is measured relatively to a horizontal surface, i.e. sun on the horizon $=0^{\circ}$, sun at its zenith $=90^{\circ}$

- The output of shadowHeight and inShadow is a numeric or logical "matrix", respectively, where rows represent locations and columns represent solar positions. The output of shadowFootprint is a polygonal layer of footprints. The output of SVF is a numeric vector where values correspond to locations. All functions that can accept a raster of ground locations return a corresponding raster of computed values

The "high-level" function radiation is a wrapper around inShadow and SVF for calculating direct and diffuse solar radiation on the obstacle surface area (i.e. building roofs and facades). In addition to the geometric layers and solar positions, this function also requires meteorological measurements of direct and diffuse radiation at an unobstructed weather station. The shadow package provides a sample Typical Meteorological Year (TMY) dataset tmy to illustrate the usage of the radiation function (see below). Similar TMY datasets were generated for many areas (e.g. Pusat et al., 2015) and are generally available from meteorological agencies, or from databases for building energy simulation such as EnergyPlus (?).

Finally, the shadow package provides several "helper functions" which are used internally by "low-level" and "high-level" functions, but can also be used independently -

- classifyAz - Determines the azimuth where the perpendicular of a line segment is facing; used internally to classify facade azimuth

- coefDirect - Calculates the Coefficient of Direct Normal Irradiance reduction (Equations 5 and 6)

- plotGrid - Makes an interactive plot of 3D spatial points. This is a wrapper around scatterplot3js from package threejs (Lewis, 2017)

- ray - Creates a spatial line between two given points

- shiftAz - Shifts spatial features by azimuth and distance

- surfaceGrid - Creates a 3D point layer with a grid which covers the facades and roofs of obstacles

- toSeg - Splits polygons or lines to segments

The following section provides a manual for using these functions through a simple example with four buildings.

\footnotetext{
${ }^{2}$ The third dimension of 3D points has to be specified using three-dimensional coordinates, rather than a "height" attribute in a 2D point layer (see Examples section)
} 


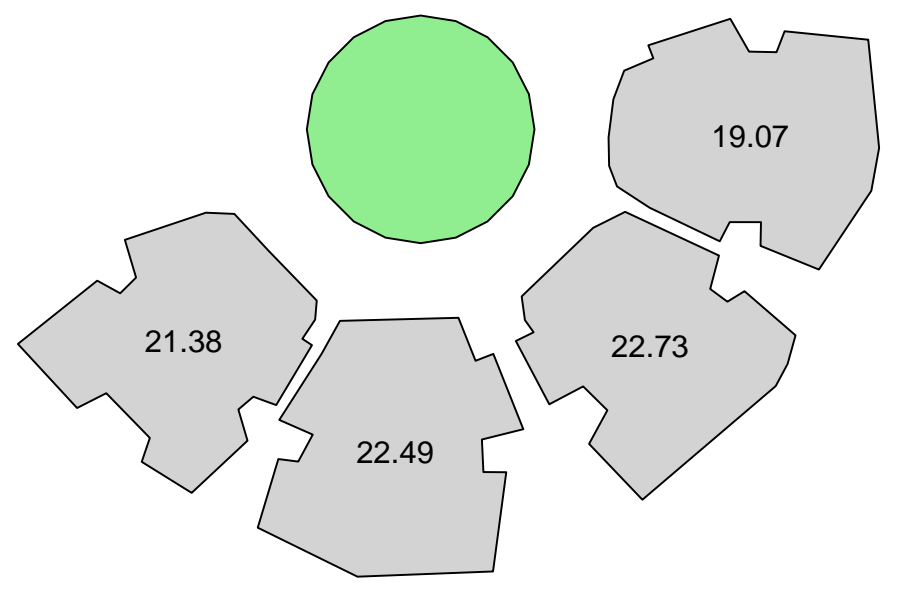

Figure 6: Sample data: a buildings layer and a green park layer. Text labels express building height in meters.

\section{Examples}

In this section we demonstrate the main functionality of shadow, namely calculating -

- Shadow height (function shadowHeight)

- Logical shadow flag (function inShadow)

- Shadow footprint (function shadowFootprint)

- Sky View Factor (function SVF)

- Solar radiation (function radiation)

Before going into the examples, we load the shadow package. Package sp is loaded automatically along with shadow. Packages raster (Hijmans, 2017) and rgeos (Bivand and Rundel, 2017) are used throughout the following code examples for preparing the inputs and presenting the results, so they are loaded as well.

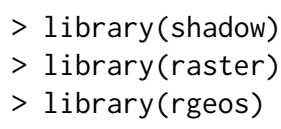

In the examples, we will use a small real-life dataset representing four buildings in Rishon-Le-Zion, Israel (Figure 6), provided with package shadow and named build.

The following code section also creates a hypothetical circular green park located 20 meters to the north and 8 meters to the west from the buildings layer centroid (hereby named park).

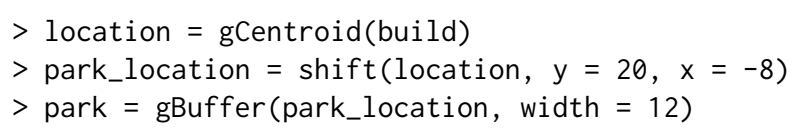

The following expressions visualize the build and park layers as shown in Figure 6. Note that the build layer has an attribute named BLDG_HT specifying the height of each building (in meters), as shown using text labels on top of each building outline.

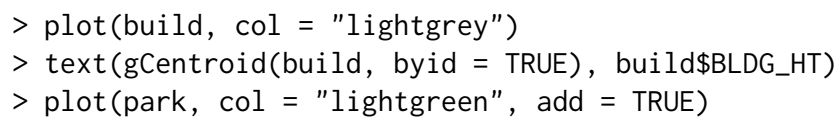

\section{Shadow height}

The shadowHeight function calculates shadow height(s) at the specified point location(s), given a layer of obstacles and solar position(s). The shadowHeight function, as well as other functions that require a solar position argument such as inShadow, shadowFootprint and radiation (see below), alternatively accept a time argument instead of the solar position. In case a time (time) argument is passed instead of solar position (solar_pos), the function internally calculates solar position using the lon/lat of 
the location layer centroid and the specified time, using function solarpos from package maptools (Bivand and Lewin-Koh, 2017).

In the following example, we would like to calculate shadow height at the centroid of the buildings layer (build) on 2004-12-24 at 13:30:00. First we create the queried points layer (location), in this case consisting of a single point: the build layer centroid. This is our layer of locations where we would like to calculate shadow height.

$>$ location = gCentroid(build)

Next we need to specify the solar position, i.e. sun elevation and azimuth, at the particular time and location $\left(31.967^{\circ} \mathrm{N} 34.777^{\circ} \mathrm{E}\right)$, or let the function calculate it automatically based on the time. Using the former option, we can figure out solar position using function solarpos from package maptools. To do that, we first define a "POSIXct" object specifying the time we are interested in -

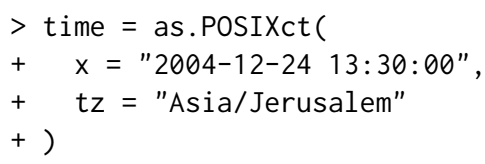

Second, we find the longitude and latitude of the point by reprojecting it to a geographic $\mathrm{CRS}^{3}$.

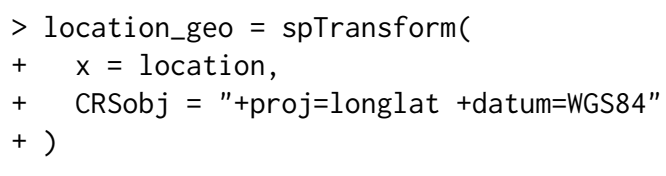

Finally, we use the solarpos function to find solar position, given longitude, latitude and time -

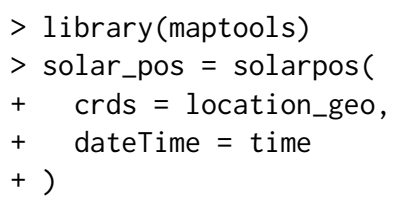

We now know the sun azimuth $\left(208.7^{\circ}\right)$ and elevation $\left(28.8^{\circ}\right)$ -

$>$ solar_pos

$$
\begin{aligned}
& \text { \#> } \quad[, 1] \quad[, 2] \\
& \text { \#> }[1,] 208.733328 .79944
\end{aligned}
$$

Given the solar position along with the layer of obstacles build, shadow height in location can be calculated using the shadowHeight function, as follows -

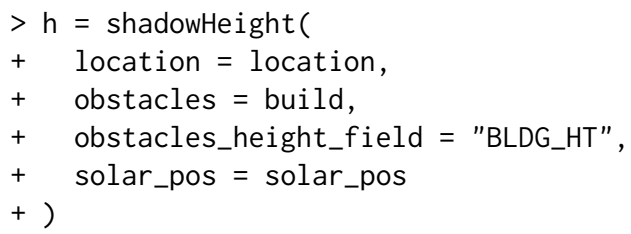

The resulting object contains the shadow height value of 19.86 meters -

$$
\begin{aligned}
& >\mathrm{h} \\
& \text { \#> } \quad[, 1] \\
& \text { \#> }[1,] 19.86451
\end{aligned}
$$

The second (shorter) approach is letting the function calculate solar position for us, in which case we can pass just the spatial layers and the time, without needing to calculate solar position ourselves -

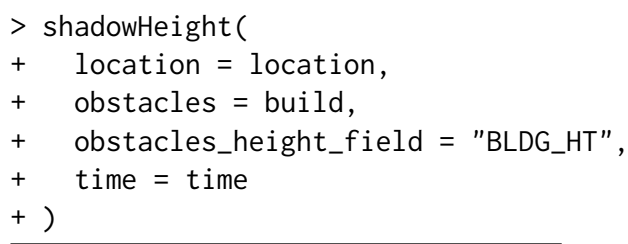

${ }^{3}$ Note that calculating solar position is the only example where lon/lat coordinates are needed when working with shadow. All other spatial inputs are required to be passed in a projected CRS, due to the fact that obstacles height is meaningless to specify in lon/lat degree units 


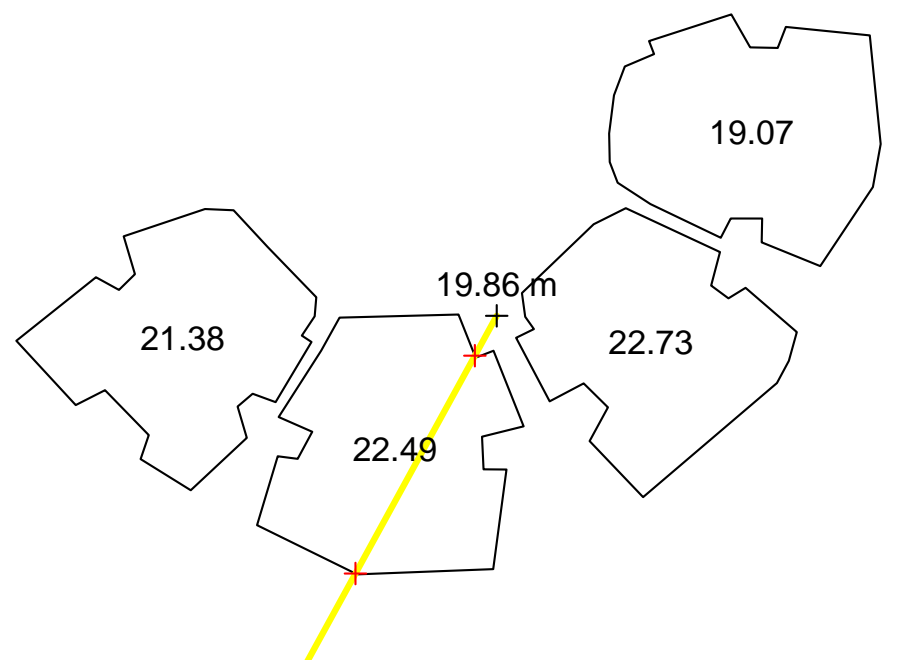

Figure 7: Shadow height $(m)$ at a single point (indicated by black + symbol)

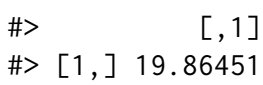

The results of both approaches are identical. The first approach, where solar position is manually defined, takes more work and thus may appear unnecessary. However, it is useful for situations when we want to use specific solar positions from an external data source, or to evaluate arbitrary solar positions that cannot be observed in the queried location in real life.

Either way, the resulting object $h$ is a "matrix", though in this case it only has a single row and a single column. The shadowHeight function accepts location layers with more than one point, in which case the resulting "matrix" will have additional rows. It also accepts more than one solar position or time value (see below), in which case the resulting "matrix" will have additional columns. It is thus possible to obtain a matrix of shadow height values for a set of locations in a set of times.

Figure 7 illustrates how the shadow height calculation was carried out. First, a line of sight is drawn between the point of interest and the sun direction based on sun azimuth (shown as a yellow line). Next, potential intersections are detected (marked with + symbols). Finally, shadow height induced by each intersection is calculated based on the distance towards intersection, sun elevation and intersected building height (see Figure 1). The final result is the maximum of the per-intersection heights.

The procedure can be readily expanded to calculate a continuous surface of shadow heights, as the shadowHeight function also accepts "Raster*" objects (package raster). The raster serves as a template, defining the grid where shadow height values will be calculated. For example, in the following code section we create such a template raster covering the examined area plus a 50-meter buffer on all sides, with a spatial resolution of 2 meters -

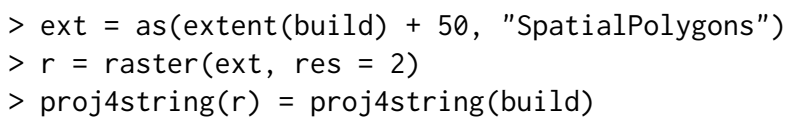

Now we can calculate a shadow height raster by simply replacing the location argument with the raster $r$ -

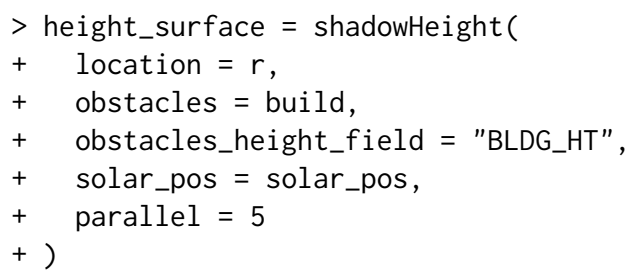

The result (height_surface), in this case, is not a matrix - it is a shadow height surface (a "RasterLayer" object) of the same spatial dimensions as the input template $r$. Note that unshaded pixels get an NA shadow height value, thus plotted in white (Figure 8). Also note the partial shadow on the roof of the north-eastern building (top-right) caused by the neighboring building to the south-west. 


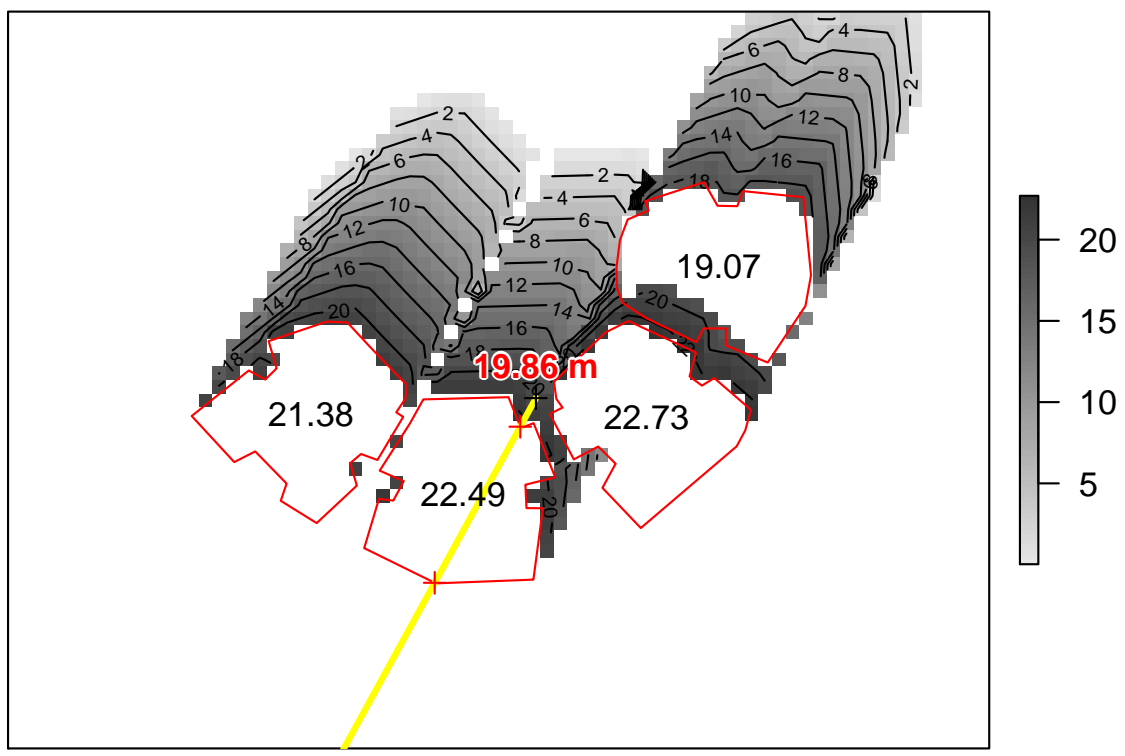

Figure 8: Shadow height $(m)$ surface, and an individual shadow height value (indicated by black + symbol at the center of the image)

The additional parallel $=5$ argument splits the calculation of raster cells among 5 processor cores, thus making it faster. A different number can be specified, depending the number of available cores. Behind the scenes, parallel processing relies on the parallel package (R Core Team, 2018).

\section{Shadow (logical)}

Function shadowHeight, introduced in the previous section, calculates shadow height for a given ground location. In practice, the metric of interest is very often whether a given 3D location is in shade or not. Such a logical flag can be determined by comparing the Z-coordinate (i.e. the height) of the queried point with the calculated shadow height at the same $X-Y$ location. The inShadow function is a wrapper around shadowHeight for doing that.

The inshadow function gives the logical shadow/non-shadow classification for a set of 3D points. The function basically calculates shadow height for a given unique ground location $(X-Y)$, then compares it with the elevation $(Z)$ of all points in that location. The points which are positioned "above" the shadow are considered non-shaded (receiving the value of FALSE), while the points which are positioned "below" the shadow are considered shaded (receiving the value of TRUE).

The 3D points we are interested in when doing urban analysis are usually located on the surface of elements such as buildings. The surfaceGrid helper function can be used to automatically generate a grid of such surface points. The inputs for this function include the obstacle layer for which to generate a surface grid and the required grid resolution. The returned object is a 3D point layer.

For example, the following expression calculates a $3 \mathrm{D}$ point layer named grid covering the build surface at a resolution of 2 meters -

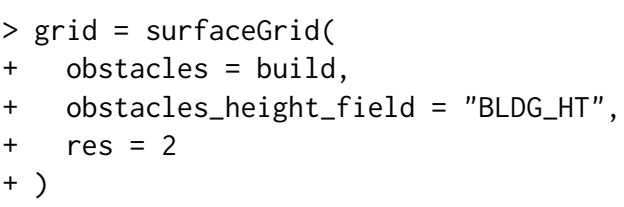

The resulting grid points are associated with all attributes of the original obstacles each surface point corresponds to, as well as six new attributes -

- obs_id - Unique consecutive ID for each feature in obstacles

- type - Either "facade" or "roof"

- seg_id - Unique consecutive ID for each facade segment (only for "facade" points)

- xy_id - Unique consecutive ID for each ground location (only for "facade" points)

- facade_az - The azimuth of the corresponding facade, in decimal degrees (only for "facade" points)

In this case, the resulting 3D point grid has 2,693 features, starting with "roof" points - 


> head(grid)
$\begin{array}{lrrrrrrr}\text { \#> } & \text { build_id } & \text { BLDG_HT } & \text { obs_id type } & \text { seg_id } & \text { xy_id } & \text { facade_az } \\ \text { \#> } & 7 & 722 & 22.49 & 3 \text { roof } & \text { NA } & \text { NA } & \text { NA } \\ \text { \#> } 2 & 722 & 22.49 & 3 \text { roof } & \text { NA } & \text { NA } & \text { NA } \\ \text { \#> } & 722 & 22.49 & 3 \text { roof } & \text { NA } & \text { NA } & \text { NA } \\ \text { \#> } & 72 & 722 & 22.49 & 3 \text { roof } & \text { NA } & \text { NA } & \text { NA } \\ \text { \#> 5 } & 722 & 22.49 & 3 \text { roof } & \text { NA } & \text { NA } & \text { NA } \\ \text { \#> } 6 & 722 & 22.49 & \text { 3 roof } & \text { NA } & \text { NA } & \text { NA }\end{array}$

Then going through the "facade" points -

$>\operatorname{tail}($ grid)

$\begin{array}{lrrrrrr}\text { \#> } & \text { build_id } & \text { BLDG_HT } & \text { obs_id } & \text { type seg_id } & \text { xy_id } & \text { facade_az } \\ \text { \#> } 19610 & 831 & 19.07 & \text { 4 facade } & 74 & 44 & 100.2650 \\ \text { \#> } 19710 & 831 & 19.07 & \text { 4 facade } & 75 & 45 & 123.6695 \\ \text { \#> } 19810 & 831 & 19.07 & 4 \text { facade } & 75 & 46 & 123.6695 \\ \text { \#> } 19910 & 831 & 19.07 & 4 \text { facade } & 75 & 47 & 123.6695 \\ \text { \#> } 20010 & 831 & 19.07 & 4 \text { facade } & 75 & 48 & 123.6695 \\ \text { \#> } 20110 & 831 & 19.07 & \text { 4 facade } & 75 & 49 & 123.6695\end{array}$

Printing the coordinates confirms that, indeed, grid is a 3D point layer having three-dimensional coordinates where the third dimension $\mathrm{h}$ represents height above ground -

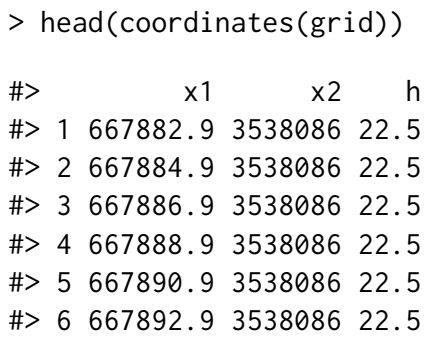

Once the 3D grid is available, we can evaluate whether each point is in shadow or not, at the specified solar position(s), using the inShadow wrapper function -

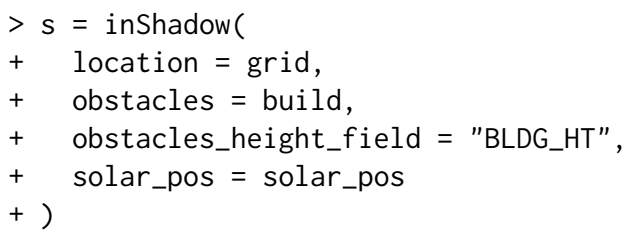

The resulting object $s$ is a "logical" matrix with rows corresponding to the grid features and columns corresponding to the solar positions. In this particular case a single solar position was evaluated, thus the matrix has just one column -

$>\operatorname{dim}(\mathrm{s})$

\#> [1] $2693 \quad 1$

The scatter 3D function from package plot3D (Soetaert, 2017) is useful for visualizing the result. In the following code section, we use two separate scatter3D function calls to plot the grid with both variably colored filled circles (yellow or grey) and constantly colored (black) outlines.

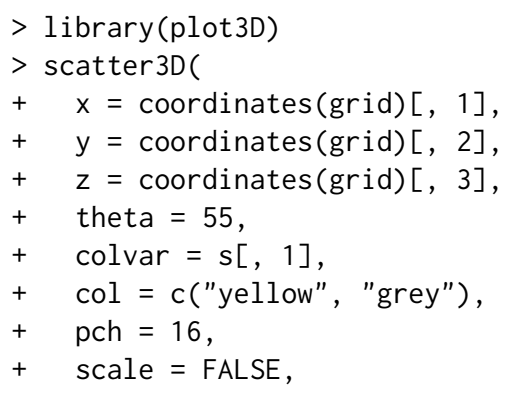




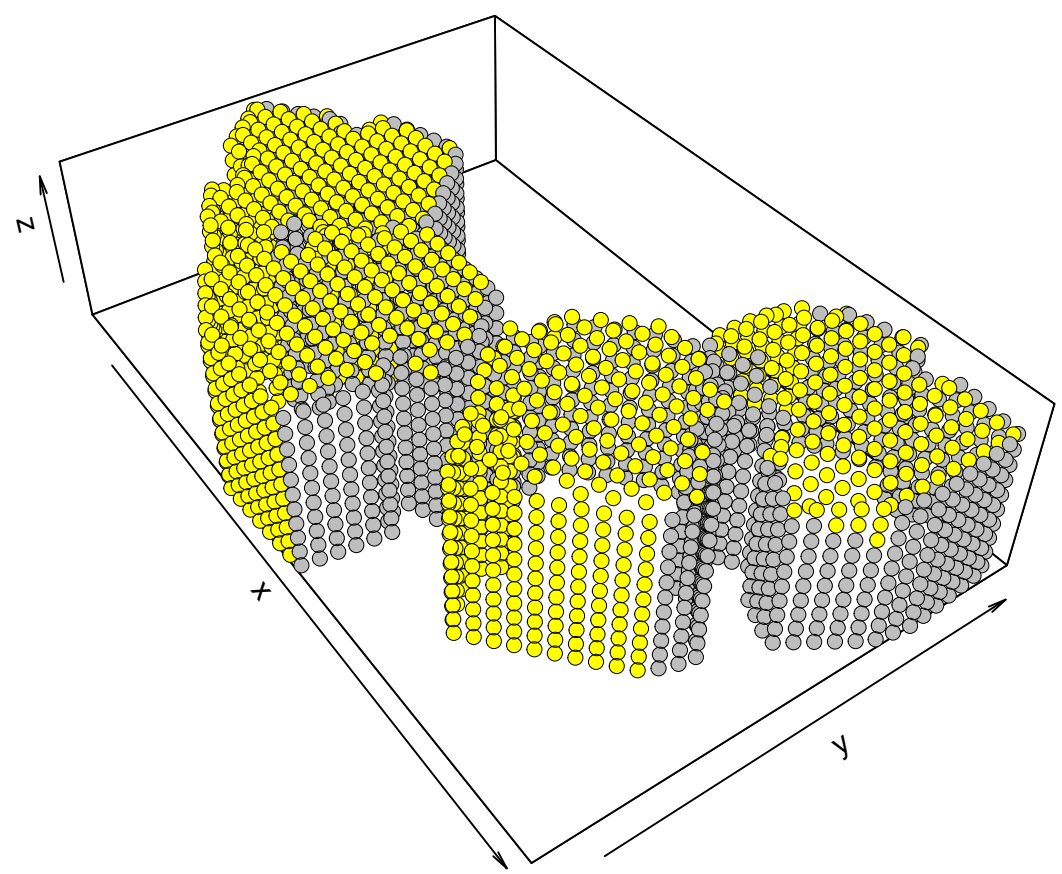

Figure 9: Buildings surface points in shadow (grey) and in direct sunlight (yellow) on 2004-12-24 13:30:00

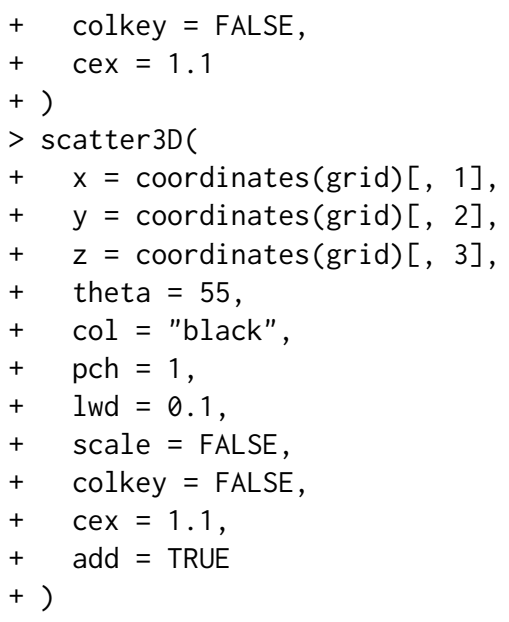

The output is shown in Figure 9. It shows the 3D grid points, along with the inShadow classification encoded as point color: grey for shaded surfaces, yellow for sun-exposed surfaces.

\section{Shadow footprint}

The shadowFootprint function calculates the geometry of shadow projection on the ground. The resulting footprint layer can be used for various applications. For example, a shadow footprint layer can be used to calculate the proportion of shaded surface in a defined area, or to examine which obstacles are responsible for shading a given urban element.

In the following example, the shadowFootprint function is used to determine the extent of shading on the hypothetical green park (Figure 6) at different times of day. First, let us consider a single time instance of 2004-06-24 09:30:00. At this particular time and geographical location, the solar position is at an azimuth of $88.8^{\circ}$ and at an elevation of $46.7^{\circ}$

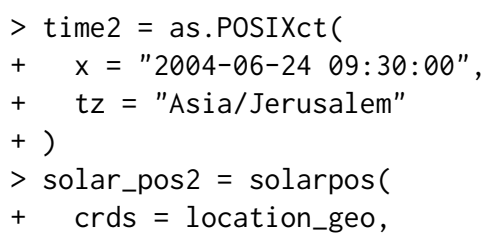




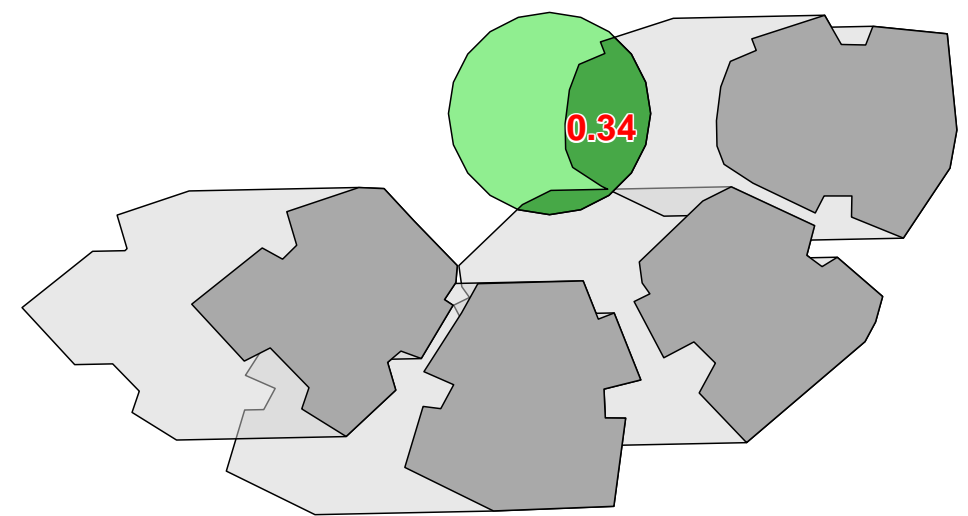

Figure 10: Shaded park proportion on 2004-06-24 09:30:00

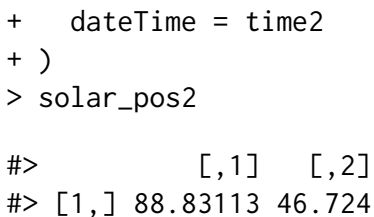

The following expression calculates the shadow footprint for this particular solar position.

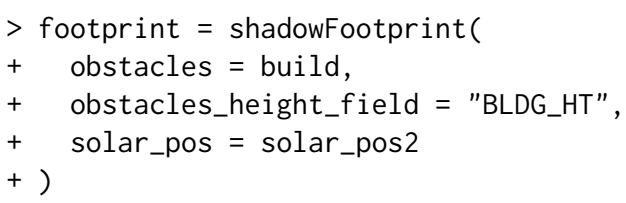

The resulting object footprint is a polygonal layer ("SpatialPolygonsDataFrame" object) which can be readily used in other spatial calculations. For example, the footprint and park polygons can be intersected to calculate the proportion of shaded park area within total park area, as follows.

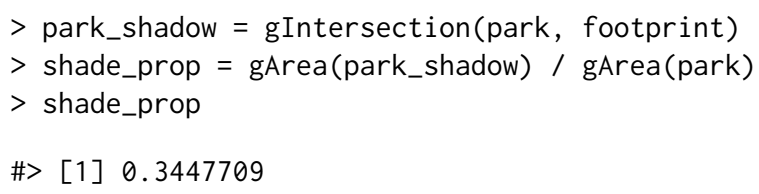

The numeric result shade_prop gives the proportion of shaded park area, 0.34 in this case (Figure

The shadow footprint calculation can also be repeated for a sequence of times, rather than a single one, to monitor the daily (monthly, annual, etc.) course of shaded park area proportion. To do that, we first need to prepare the set of solar positions in the evaluated dates/times. Again, this can be done using function solarpos. For example, the following code creates a matrix named solar_pos_seq containing solar positions over the 2004-06-24 at hourly intervals -

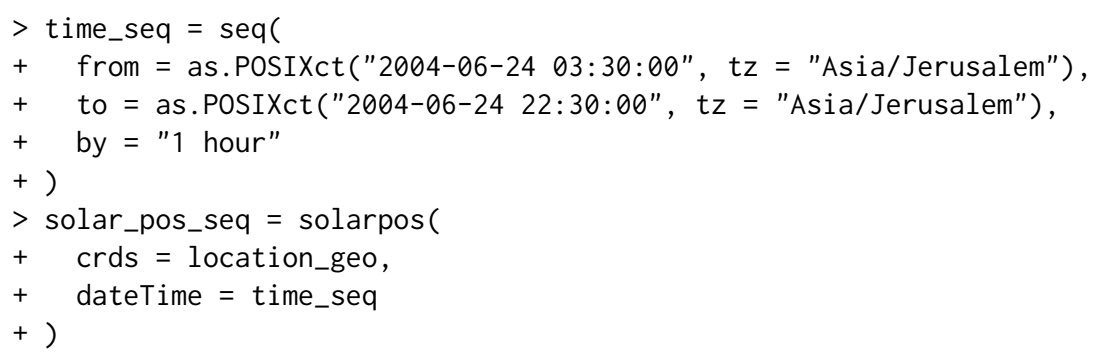

Note that the choice of an hourly interval is arbitrary. Shorter intervals (e.g. 30 mins) can be used for increased accuracy.

To calculate the shaded park proportion at each time step we can loop over the solar_pos_seq matrix, each time -

- Calculating shadow footprint 


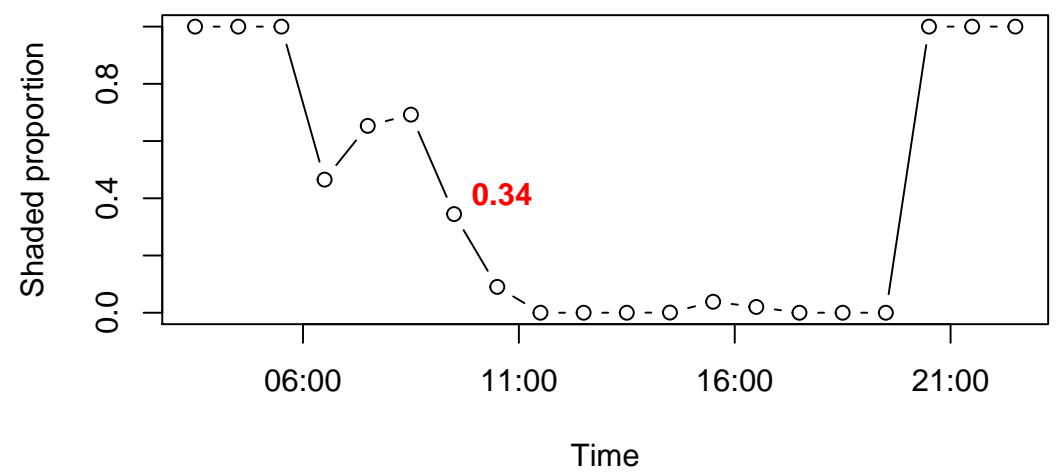

Figure 11: Shaded park proportion at each hourly time step on 2004-06-24

- Intersecting the shadow footprint with the park outline

- Calculating the ratio of intersection and total park area

The code of such a for loop is given below.

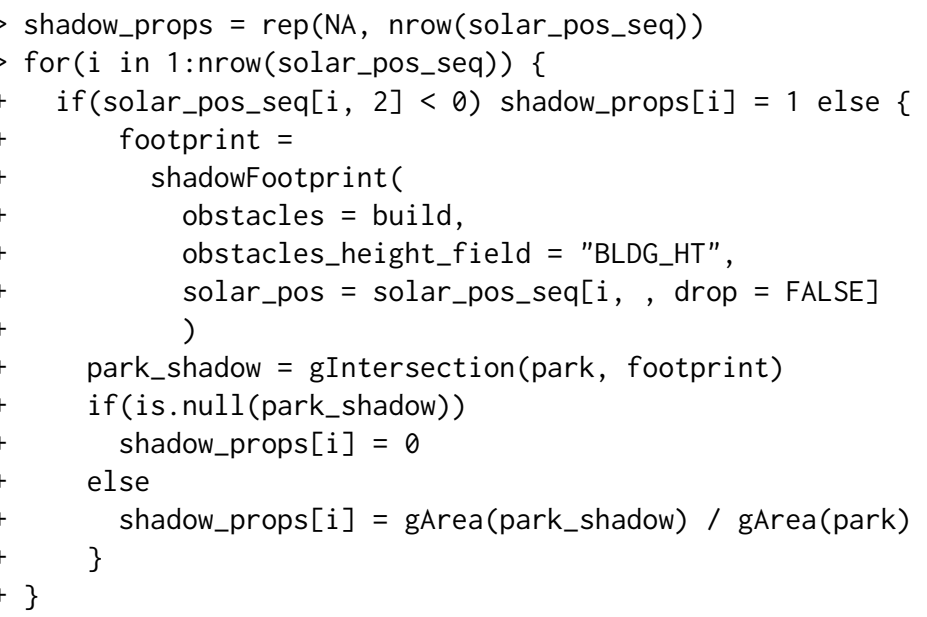

The loop creates a numeric vector named shadow_props. This vector contains shaded proportions for the park in agreement with the times we specified in time_seq. Note that two conditional statements are being used to deal with special cases -

- Shadow proportion is set to 1 (i.e. maximal) when sun is below the horizon

- Shadow proportion is set to 0 (i.e. minimal) when no intersections are detected between the park and the shadow footprint

Plotting shadow_props as function of time_seq (Figure 11) summarizes the daily course of shaded park proportion on the 2004-06-24. The individual value of 0.34 which we have calculated for 09:30 in the previous example (Figure 10) is highlighted in red.

\section{Sky View Factor}

The SVF function can be used to estimate the SVF at any 3D point location. For example, the following expression calculates the SVF on the ground ${ }^{4}$ at the centroid of the build layer (Figure 4).

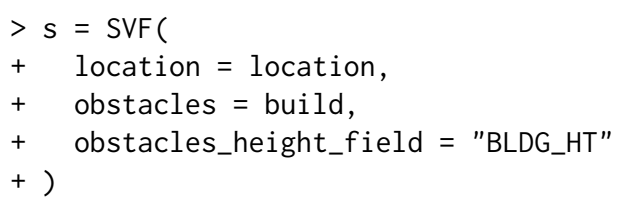

The resulting SVF is 0.396 , meaning that about $39.6 \%$ of the sky area are visible (Figure 12) from this particular location.

\footnotetext{
${ }^{4}$ Recall (Table 1) that the inShadow and SVF functions accept either 2D or 3D points, whereas 2D points are treated as ground locations
} 


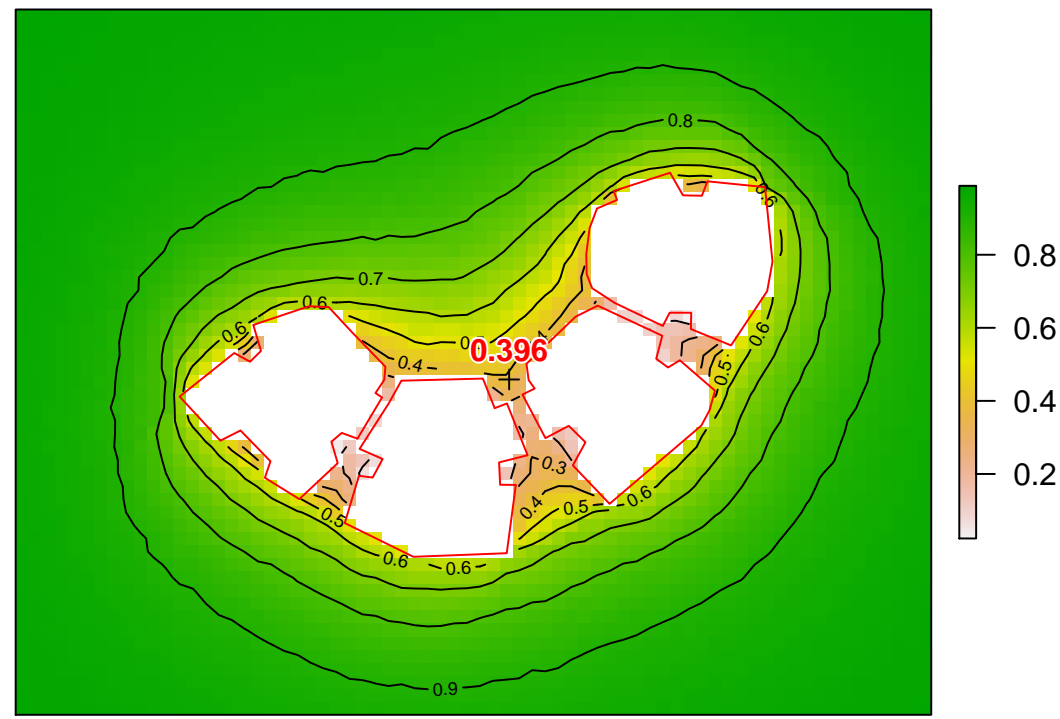

Figure 12: Sky View Factor (SVF) surface, with SVF value for an individual point (indicated by black + symbol at the center of the image)

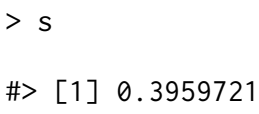

Note that the SVF function has a tuning parameter named res_angle which can be used to modify angular resolution (default is $5^{\circ}$, as shown in Figure 4). A smaller res_angle value will give more accurate SVF but slower calculation.

Given a "template" grid, the latter calculation can be repeated to generate a continuous surface of SVF estimates for a grid of ground locations. In the following code section we calculate an SVF surface using the same raster template with a resolution of 2 meters from the shadow height example (see above).

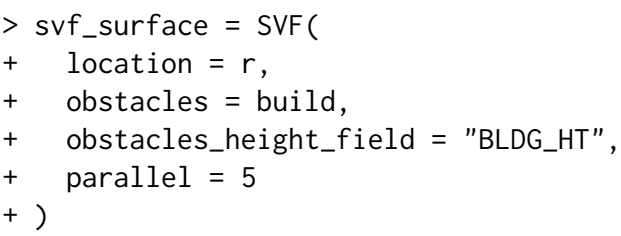

Note that the parallel=5 option is used once again to make the calculation run simultaneously on 5 cores. The resulting SVF surface is shown in Figure 12. As could be expected, SVF values are lowest in the vicinity of buildings due to their obstruction of the sky.

\section{Solar radiation}

Shadow height, shadow footprint and SVF can be considered as low-level geometric calculations. Frequently, the ultimate aim of an analysis is the estimation of insolation, which is dependent on shadow and SVF but also on surface orientation and meteorological solar radiation conditions. Thus, the low-level geometric calculations are frequently combined and wrapped with meteorological solar radiation estimates to take the geometry into account when evaluating insolation over a given time interval. The shadow package provides this kind of wrapper function named radiation.

The radiation function needs several parameters to run -

- 3D points grid representing surfaces where the solar radiation is evaluated. It is important to specify whether each grid point is on a "roof" or on a "facade", and the azimuth it is facing (only for "facade"). A grid with those attributes can be automatically produced using the surfaceGrid function (see above)

- Obstacles layer defined with obstacles, having an obstacles_height_field attribute (see above)

- Solar positions defined with solar_pos (see above) 
- Meteorological estimates defined with solar_normal and solar_diffuse, corresponding to the same time intervals given by solar_pos

Given this set of inputs, the radiation function:

- calculates whether each grid surface point is in shadow or not, for each solar position solar_pos, using the inshadow function (Equation 1),

- calculates the Coefficient of Direct Normal Irradiance reduction, for each grid surface point at each solar position solar_pos, using the coefDirect function (Equations 5 and 6),

- combines shadow, the coefficient and the meteorological estimate solar_normal to calculate the direct radiation (Equation 7),

- calculates the SVF for each grid surface point, using the SVF function (Equations 3 and 4),

- combines the SVF and the meteorological estimate solar_diffuse to calculate the diffuse radiation (Equation 8)

- and calculates the sums of the direct, diffuse and total (i.e. direct+diffuse) solar radiation per grid surface point for the entire period (Equation 9).

To demonstrate the radiation function, we need one more component not used in the previous examples: the reference solar radiation data. The shadow package comes with a sample Typical Meteorological Year (TMY) dataset named tmy that can be used for this purpose. This dataset was compiled for the same geographical area where the buildings are located, and therefore can be realistically used in our example.

The tmy object is a data. frame with 8,760 rows, where each row corresponds to an hourly interval over an entire year $(24 \times 365=8,760)$. The attributes given for each hourly interval include solar position (sun_az, sun_elev) and solar radiation measurements (solar_normal, solar_diffuse). Both solar radiation measurements are given in $W / m^{2}$ units.

\begin{tabular}{|c|c|c|c|c|c|c|c|}
\hline \#> & & time & sun_az & sun_elev & solar_normal & solar_diffuse & dbt ws \\
\hline \#> 1 & $1999-01-01$ & $01: 00: 00$ & 66.73 & -70.94 & 0 & 0 & 6.61 .0 \\
\hline$\#>2$ & $1999-01-01$ & $02: 00: 00$ & 82.02 & -58.68 & 0 & 0 & 5.91 .0 \\
\hline$\#>3$ & $1999-01-01$ & $03: 00: 00$ & 91.00 & -45.99 & 0 & 0 & 5.41 .0 \\
\hline$\#>4$ & $1999-01-01$ & $04: 00: 00$ & 98.13 & -33.32 & 0 & 0 & 4.91 .0 \\
\hline$\#>5$ & $1999-01-01$ & $05: 00: 00$ & 104.81 & -20.86 & 0 & 0 & 4.41 .0 \\
\hline$\#>6$ & $1999-01-01$ & $06: 00: 00$ & 111.73 & -8.76 & 0 & 6 & 4.81 .0 \\
\hline$\#>7$ & $1999-01-01$ & $07: 00: 00$ & 119.41 & 2.91 & 118 & 24 & 7.31 .0 \\
\hline$\#>8$ & $1999-01-01$ & $08: 00: 00$ & 128.39 & 13.30 & 572 & 45 & 11.21 .0 \\
\hline$\#>9$ & $1999-01-01$ & $09: 00: 00$ & 139.20 & 22.46 & 767 & 57 & 16.01 .0 \\
\hline \#> 10 & $1999-01-01$ & $10: 00: 00$ & 152.33 & 29.63 & 809 & 66 & 16.32 .1 \\
\hline
\end{tabular}

The Direct Normal Irradiance (solar_normal) is the amount of solar radiation received per unit area by a surface that is always held normal to the incoming rays from the sun's current position in the sky. This is an estimate of maximal direct radiation, obtained on an optimally tilted surface. The Diffuse Horizontal Irradiance (solar_diffuse) is the amount of radiation received per unit area at a surface that has not arrived on a direct path from the sun, but has been scattered by molecules and particles in the atmosphere. This is an estimate of diffuse radiation.

To use the solar positions from the tmy dataset, we create a separate matrix with just the sun_az and sun_elev columns -

$>$ solar_pos = as.matrix (tmy[, c("sun_az", "sun_elev")])

The first few rows of this matrix are -

\begin{tabular}{lrr}
\multicolumn{4}{l}{ head(solar_pos) } \\
$\begin{array}{lrr}\text { \#> } & \text { sun_az } & \text { sun_elev } \\
\text { \#> } 2 & 66.73 & -70.94 \\
\text { \#> } 3 & 82.02 & -58.68 \\
\text { \#> } 4 & 91.00 & -45.99 \\
\text { \#> } 5 & 98.13 & -33.32 \\
\text { \#> } 6 & 104.81 & -20.86 \\
\text { \#>7 } & 111.73 & -8.76\end{array}$
\end{tabular}


Now we have everything needed to run the radiation function. We are hereby using the same grid layer with 3D points covering the roofs and facades of the four buildings created above using the surfaceGrid function (Figure 9), the layer of obstacles, and the solar position and measured solar radiation at a reference weather station from the tmy table.

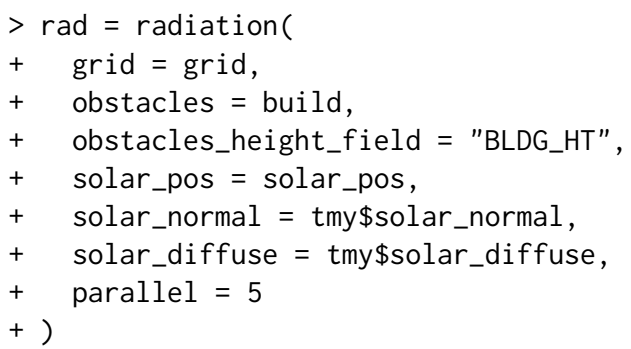

The returned object rad is a data. frame with the summed direct, diffuse and total (i.e. direct+diffuse) solar radiation estimates, as well as the SVF, for each specific surface location in grid. Summation takes place over the entire period given by solar_pos, solar_normal and solar_diffuse. In the present case it is an annual insolation. The units of measurement are therefore $\mathrm{Wh} / \mathrm{m}^{2}$ summed over an entire year.

For example, the following printout -

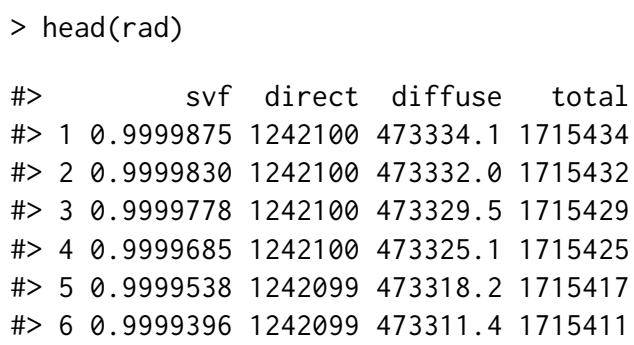

refers to the first six surface points which are part of the same roof, thus sharing similar annual solar radiation estimates. Overall, however, the differences in insolation are very substantial among different locations on the buildings surfaces, as shown in Figure 13. For example, the roofs receive about twice as much direct radiation as the south-facing facades. The code for producing Figure 13, using function scatter 3D (see Figure 9), can be found on the help page of the radiation function and is thus omitted here to save space. Note that the figure shows radiation estimates in $\mathrm{kWh} / \mathrm{m}^{2}$ units, i.e. the values from the rad table (above) divided by 1000.

\section{Discussion}

The shadow package introduces a simple geometric model for shadow-related calculations in an urban environment. Specifically, the package provides functions for calculating shadow height, shadow footprint and SVF. The latter can be combined with TMY data to estimate insolation on built surfaces. It is, to the best of our knowledge, the only $\mathrm{R}$ package aimed at shadow calculations in a vector-based representation of the urban environment. It should be noted that the insol package provides similar functionality for a raster-based environment, but the latter is more suitable for modelling large-scale natural environments rather than detailed urban landscapes.

The unique aspect of our approach is that calculations are based on a vector layer of polygons extruded to a given height, known as $2.5 \mathrm{D}$, such as building footprints with a height attribute. The vector-based 2.5D approach has several advantages over the two commonly used alternative ones: vector-based $3 \mathrm{D}$ and raster-based models. Firstly, the availability of $2.5 \mathrm{D}$ input data is much greater compared to both specialized 3D models and high-resolution raster surfaces. Building layers for entire cities are generally available from various sources, ranging from local municipality GIS systems to global crowd-sourced datasets (e.g. OpenStreetMap) (Haklay and Weber, 2008). Secondly, processing does not require closed-source software, or interoperability with complex specialized software, as opposed to working with 3D models. Thirdly, results are easily associated back to the respective urban elements such as buildings, parks, roofs facades, etc., as well as their attributes, via a spatial join operation (e.g. using function over in R package sp). For example, we can easily determine which building is responsible for shading the green park in the above shadow footprint example (Figure 10). This is unlike a raster-based approach, where the input is a continuous surface with no attributes, thus having no natural association to individual urban elements or objects. 

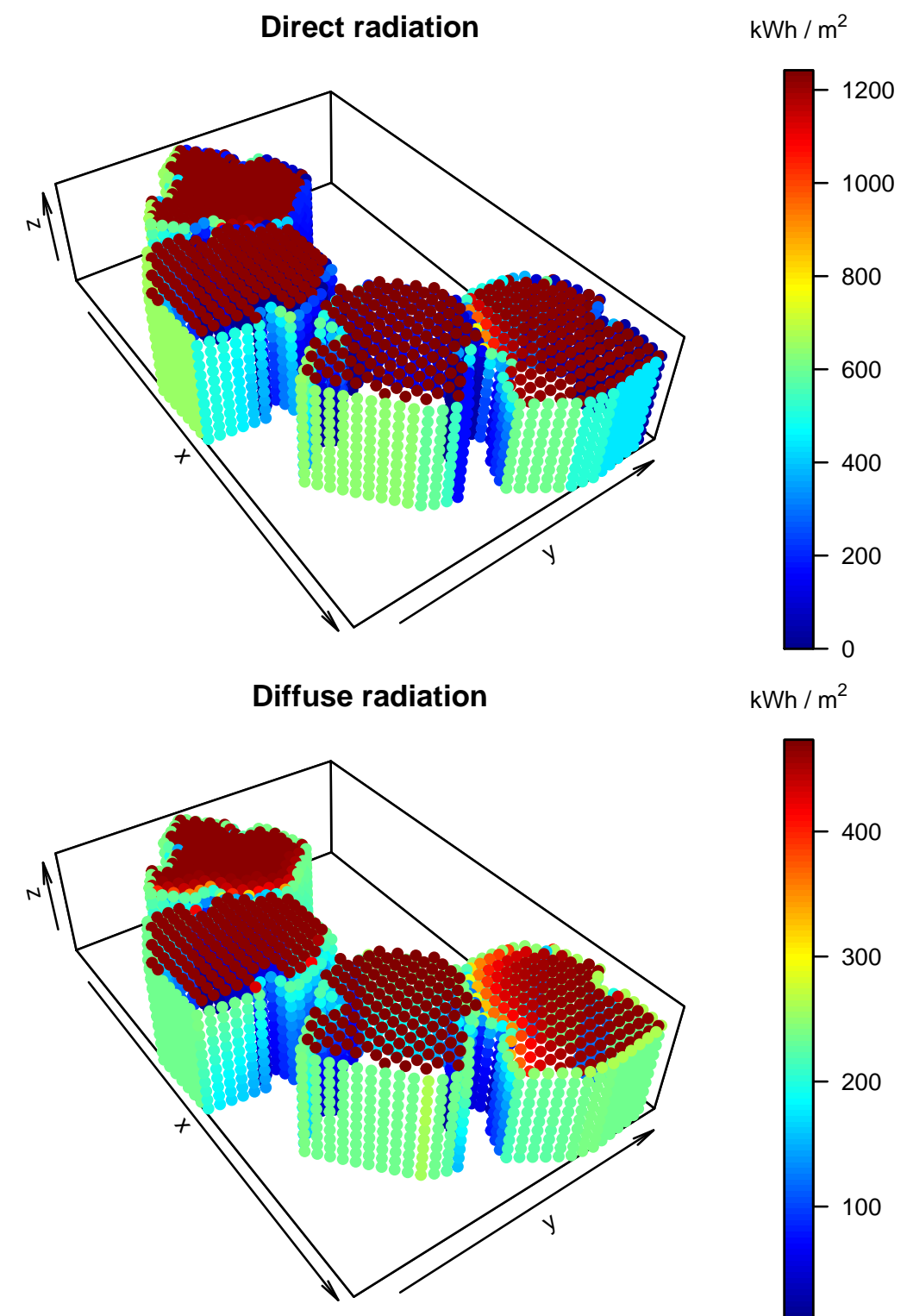

$\mathrm{kWh} / \mathrm{m}^{2}$
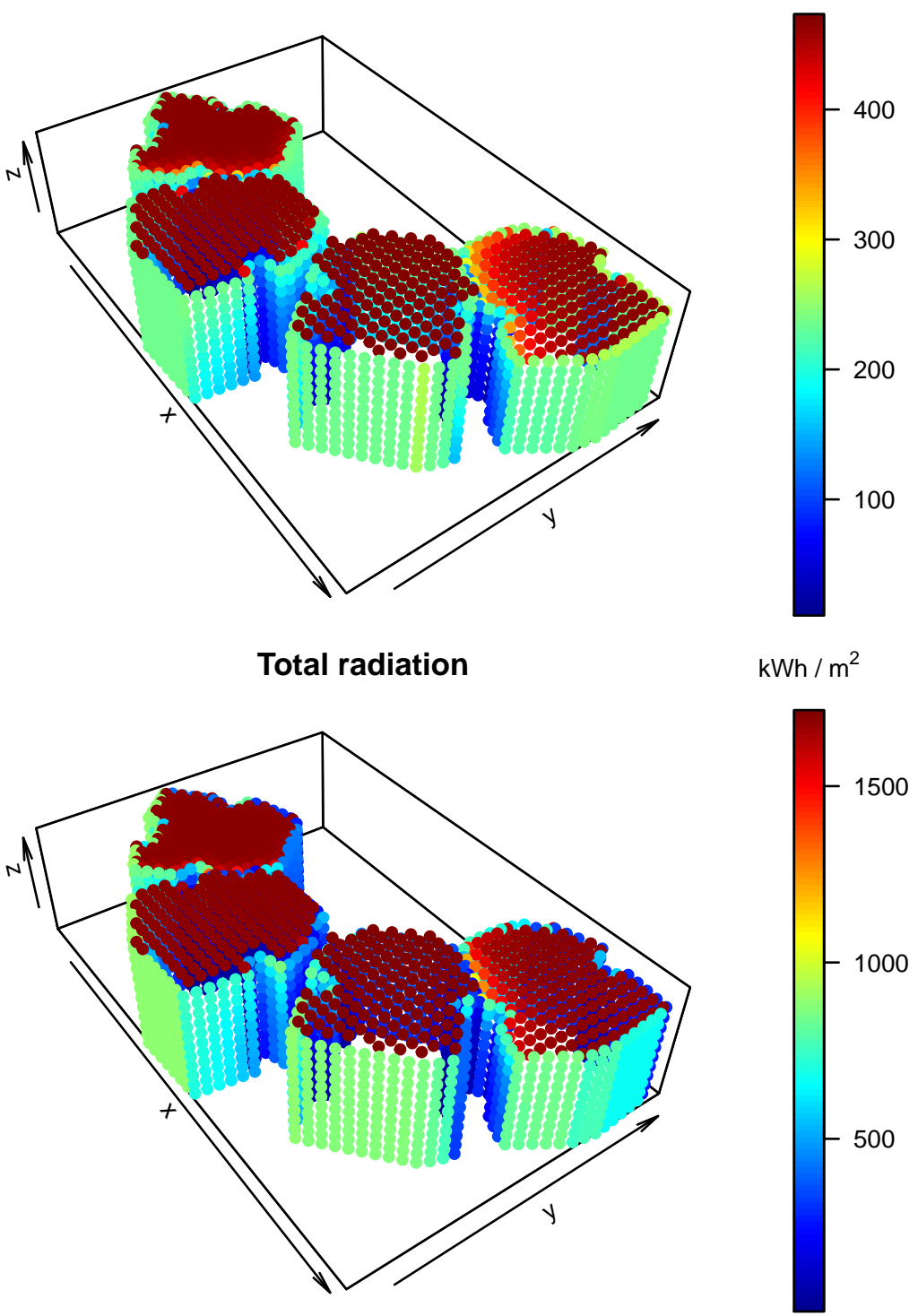

Figure 13: Annual direct, diffuse and total radiation estimates per grid point $\left(\mathrm{kWh} / \mathrm{m}^{2}\right)$. Note that the Y-axis points to the north. Also note that the color scale range is different in each panel. 
However, it should be noted that the $2.5 \mathrm{D}$ vector-based approach requires several assumptions and has some limitations. When the assumptions do not hold, results may be less accurate compared to the above-mentioned alternative approaches. For example, it is impossible to represent geometric shapes that are not a simple extrusion in 2.5D (though, as mentioned above, urban surveys providing such detailed data are not typically available). An ellipsoid tree, a bridge with empty space underneath, a balcony extruding outwards from a building facade, etc., can only be represented with a polyhedral surface in a full vector-based 3D environment (Gröger and Plümer, 2012; Biljecki et al., 2016). Recently, classes for representing true-3D urban elements, such as the Simple Feature type POL YHEDRALSURFACE, have been implemented in R package sf (Pebesma, 2018). However, functions for working with those classes, such as calculating 3D-intersection, are still lacking. Implementing such functions in R could bring new urban analysis capabilities to the R environment in the future, in which solar analysis of 3D city models probably comprises a major use case (Biljecki et al., 2015).

It should also be noted that a vector-based calculation may be generally slower than a raster based calculation. This becomes important when the study area is very large. Though the present algorithms can be optimized to some extent, they probably cannot compete with raster-based calculations where sun ray intersections can be computed using fast ray-tracing algorithms based on matrix input (Amanatides et al., 1987), as opposed to computationally intensive search for intersections between a line and a polygonal layer in a vector-based environment. For example, calculating the SVF surface shown in Figure 12 requires processing 72 angular sections $\times 3,780$ raster cells $=272,160$ SVF calculations, which takes about 7.3 minutes using five cores on an ordinary desktop computer (Intel® Core $^{\mathrm{TM}}$ i7-6700 CPU @ 3.40GHz $\times$ 8). The annual radiation estimate shown in Figure 13 however takes about 3.9 hours to calculate, as it requires SVF calculation for 2,693 grid points, as well as 727 ground locations $\times 8,760$ hours $=6,368,520$ shadow height calculations.

To summarize, the shadow package can be used to calculate shadow, SVF and solar radiation in an urban environment using widely available polygonal building data, inside the R environment (e.g. Vulkan et al., 2018). Potential use cases include urban environment applications such as evaluation of micro-climatic influence for urban planning, studying urban well-being (e.g. climatic comfort) and estimating photovoltaic energy production potential.

\section{Acknowledgements}

The shadow package was developed as part of a study funded by the Israel Ministry of National Infrastructures, Energy and Water Resources under research grant \# 021-11-215.

The authors would like thank the Editor and an anonymous reviewer for the review of this article and for the thoughtful comments.

\section{Bibliography}

J. Amanatides, A. Woo, and others. A fast voxel traversal algorithm for ray tracing. In Eurographics, volume 87 , pages $3-10,1987$. [p20]

B. Beckers. Solar Energy at Urban Scale. John Wiley \& Sons, 2013. [p4]

F. Biljecki, J. Stoter, H. Ledoux, S. Zlatanova, and A. Çöltekin. Applications of 3D city models: State of the art review. ISPRS International Journal of Geo-Information, 4(4):2842-2889, 2015. URL https://doi.org/10.3390/ijgi4042842. [p1,20]

F. Biljecki, H. Ledoux, and J. Stoter. An improved LOD specification for 3D building models. Computers, Environment and Urban Systems, 59:25-37, 2016. URL https://doi .org/10.1016/j . compenvurbsys . 2016.04.005. [p20]

R. Bivand and N. Lewin-Koh. Maptools: Tools for Reading and Handling Spatial Objects, 2017. URL https: //CRAN. R-project.org/package=maptools. R package version 0.9-2. [p9]

R. Bivand and C. Rundel. Rgeos: Interface to Geometry Engine - Open Source ('GEOS'), 2017. URL https://CRAN.R-project.org/package=rgeos. R package version 0.3-26. [p8]

R. S. Bivand, E. Pebesma, and V. Gomez-Rubio. Applied Spatial Data Analysis with R, Second Edition. Springer-Verlag, 2013. URL http: //www. asdar-book.org/. [p2]

F. Bourbia and F. Boucheriba. Impact of street design on urban microclimate for semi arid climate (Constantine). Renewable Energy, 35(2):343-347, 2010. URL https://doi .org/10.1016/j. renene. 2009.07.017. [p1] 
J. G. Corripio. Insol: Solar Radiation, 2014. URL https://CRAN.R-project.org/package=insol. R package version 1.1.1. [p1]

M. Dorman. Shadow: Geometric Shadow Calculations, 2019. URL https://CRAN.R-project.org/ package=shadow. R package version 0.6.0. [p2]

E. Erell, D. Pearlmutter, and T. Williamson. Urban Microclimate: Designing the Spaces between Buildings. Earthscan/James \& James Science Publishers, 2011. URL https://doi .org/10.4324/ 9781849775397. [p4]

ESRI. ArcGIS Desktop: Release 10.5. Environmental Systems Research Institute, CA, 2017. URL https://www. arcgis.com. [p1]

S. Freitas, C. Catita, P. Redweik, and M. C. Brito. Modelling solar potential in the urban environment: State-of-the-art review. Renewable and Sustainable Energy Reviews, 41:915-931, 2015. URL https: //doi.org/10.1016/j.rser.2014.08.060. [p1,6]

P. Fu and P. M. Rich. Design and implementation of the Solar Analyst: An ArcView extension for modeling solar radiation at landscape scales. In Proceedings of the Nineteenth Annual ESRI User Conference, pages 1-31, 1999. [p1]

B. Givoni. Climate Considerations in Building and Urban Design. John Wiley \& Sons, 1998. [p5]

Google. SketchUp: Release 17. Trimble Inc., CA, 2017. URL https://www. sketchup.com/. [p1]

GRASS Development Team. Geographic Resources Analysis Support System (GRASS GIS) Software, Version 7.2. Open Source Geospatial Foundation, 2017. URL http://grass. osgeo. org. [p1]

C. Grimmond, S. Potter, H. Zutter, and C. Souch. Rapid methods to estimate sky-view factors applied to urban areas. International Journal of Climatology, 21(7):903-913, 2001. URL https://doi . org/10. 1002/joc.659. [p4]

G. Gröger and L. Plümer. CityGML-Interoperable semantic 3D city models. ISPRS Journal of Photogrammetry and Remote Sensing, 71:12-33, 2012. URL https://doi .org/10.1016/j . isprsjprs.2012.04. 004. $[\mathrm{p} 2,20]$

T. Gál and J. Unger. A new software tool for SVF calculations using building and tree-crown databases. Urban Climate, 10:594-606, 2014. URL https://doi .org/10.1016/j.uclim. 2014.05.004. [p4]

M. Haklay and P. Weber. OpenStreetMap: User-generated street maps. IEEE Pervasive Computing, 7(4): 12-18, 2008. URL https://doi.org/10.1109/MPRV.2008.80. [p2,18]

R. J. Hijmans. Raster: Geographic Data Analysis and Modeling, 2017. URL https://CRAN. R-project. org/package=raster. R package version 2.6-7. [p8]

J. Hofierka and M. Suri. The solar radiation model for Open source GIS: Implementation and applications. In Proceedings of the Open Source GIS-GRASS Users Conference, volume 2002, pages 51-70, 2002. [p1]

J. Hofierka and M. Zlocha. A new 3-D solar radiation model for 3-D city models. Transactions in GIS, 16(5):681-690, 2012. URL https://doi .org/10.1111/j.1467-9671.2012.01337.x. [p2]

L. Kumar, A. K. Skidmore, and E. Knowles. Modelling topographic variation in solar radiation in a GIS environment. International Journal of Geographical Information Science, 11(5):475-497, 1997. URL https://doi.org/10.1080/136588197242266. [p1]

B. W. Lewis. Threejs: Interactive 3D Scatter Plots, Networks and Globes, 2017. URL https://CRAN. Rproject. org/package=threejs. R package version 0.3.1. [p7]

J. Liang, J. Gong, J. Zhou, A. N. Ibrahim, and M. Li. An open-source 3D solar radiation model integrated with a 3D Geographic Information System. Environmental Modelling \& Software, 64:94-101, 2015. URL https://doi.org/10.1016/j. envsoft.2014.11.019. [p1]

F. Lindberg, C. S. B. Grimmond, A. Gabey, B. Huang, C. W. Kent, T. Sun, N. E. Theeuwes, L. Järvi, H. C. Ward, I. Capel-Timms, and others. Urban Multi-Scale Environmental Predictor (UMEP): An integrated tool for city-based climate services. Environmental Modelling E Software, 99:70-87, 2018. URL https://doi.org/10.1016/j.envsoft.2017.09.020. [p1]

E. Pebesma. Simple Features for R: Standardized Support for Spatial Vector Data. The R Journal, 10(1): 439-446, 2018. URL https://journal.r-project.org/archive/2018/RJ-2018-009/index. html. [p20] 
E. Pebesma and R. S. Bivand. Classes and methods for spatial data: The sp package. R news, 5(2):9-13, 2005. URL https://CRAN.R-project.org/doc/Rnews/. [p2]

S. Pusat, İsmail Ekmekçi, and M. T. Akkoyunlu. Generation of typical meteorological year for different climates of Turkey. Renewable Energy, 75:144-151, 2015. URL https://doi .org/10.1016/j . renene. 2014.09.039. [p7]

QGIS Development Team. QGIS Geographic Information System. Open Source Geospatial Foundation, 2017. URL http://qgis. osgeo.org. [p1]

R Core Team. R: A Language and Environment for Statistical Computing. R Foundation for Statistical Computing, Vienna, Austria, 2018. URL https://www.R-project.org/. [p11]

C. Ratti and P. Richens. Raster analysis of urban form. Environment and Planning B: Planning and Design, 31(2):297-309, 2004. URL https://doi .org/10.1068/b2665. [p1]

P. Redweik, C. Catita, and M. Brito. Solar energy potential on roofs and facades in an urban landscape. Solar Energy, 97:332-341, 2013. URL https://doi .org/10.1016/j. solener. 2013.08.036. [p1,2]

K. Soetaert. plot3D: Plotting Multi-Dimensional Data, 2017. URL https://CRAN.R-project.org/ package=plot3D. R package version 1.1.1. [p12]

G. Van Rossum and F. L. Drake. The Python Language Reference Manual. Network Theory Ltd., 2011. URL http://www. network-theory.co.uk/python/language/. [p1]

A. Vulkan, I. Kloog, M. Dorman, and E. Erell. Modelling the potential for PV installation in residential buildings in dense urban areas. Energy and Buildings, 2018. URL https://doi.org/10.1016/j. enbuild.2018.03.052. [p20]

M. Weisthal. Assessment of potential energy savings in israel through climate-aware residential building design. Master's thesis, Ben-Gurion University of the Negev, Jacob Blaustein Institutes for Desert Research, Albert Katz International School for Desert Studies, 2014. URL http: //aranne5. bgu.ac.il/others/WeisthalMorel.pdf. [p3]

\section{Michael Dorman}

$B G U$

Department of Geography and Environmental Development Ben-Gurion University of the Negev, P.O.B. 653 Beer-Sheva, Israel dorman@post.bgu.ac.il

Evyatar Erell

$B G U$

The Jacob Blaustein Institutes for Desert Research

and the Department of Geography and Environmental Development

Ben-Gurion University of the Negev, P.O.B. 653 Beer-Sheva, Israel

Adi Vulkan

$B G U$

Department of Geography and Environmental Development

Ben-Gurion University of the Negev, P.O.B. 653 Beer-Sheva, Israel

Itai Kloog

$B G U$

Department of Geography and Environmental Development

Ben-Gurion University of the Negev, P.O.B. 653 Beer-Sheva, Israel 